Review

\title{
Medicinal Plant Identification using Gabor Filters and Deep Learning Techniques: A Paper Review
}

\author{
${ }^{1}$ Stephen Opoku Oppong, ${ }^{2}$ Frimpong Twum, ${ }^{2}$ James Ben Hayfron-Acquah and ${ }^{2}$ Yaw Marfo Missah \\ ${ }^{I}$ Department of ICT Education, University of Education, Winneba, Ghana \\ ${ }^{2}$ Department of Computer Science, Kwame Nkrumah University of Science and Technology, Kumasi, Ghana
}

\author{
Article history \\ Received: 03-08-2021 \\ Revised: 17-11-2021 \\ Accepted: 20-11-2021 \\ Corresponding Author: \\ Stephen Opoku Oppong \\ ICT Education Department, \\ University of Education, \\ Winneba, Ghana \\ Email: sooppong@uew.edu.gh
}

\begin{abstract}
Computer-aided identification of plants is a branch of machine learning that has become more recognized recently and proves itself as a vital tool in numerous sectors including pharmacological science, forestry and agriculture. This has essentially generated a zeal in creating automated systems for the identification of diverse species of plants. This study reviewed plant species classification relying on leaf textural features using Gabor filters and revealed that Gabor filters perform better when combined with other feature extraction methods. Therefore, this study proposes using Log-Gabor filter in the field of plant identification to improve accuracy since they overcome the drawbacks of Gabor filters which are; the maximum bandwidth of a Gabor filter is limited to approximately one octave and Gabor filters are not optimal if one is seeking broad spectral information with maximal spatial localization.
\end{abstract}

Keywords: Plant Identification, Gabor Filter, Log-Gabor Filter, Deep Learning, Texture

\section{Introduction}

Plant taxonomy studies how to find, identify, describe, classify and name plants. Many techniques have evolved towards plant taxonomy over the years. These include chemotaxonomic classification, anatomical classification and morphological classification. The morphological and anatomical are seen as a more traditional means of classification as compared to chemotaxonomy (Simpson, 2019).

Although Ghana's tropical vegetation is rich in medicinal plants, knowledge of their distribution and uses appear to be the preserve of the elderly and, particularly the herbalists (Adeniyi et al., 2018). Most of whom acquired this knowledge through oral transmission or as a result of using the plants in traditional medicine preparation. Generally, information gathered on the diversity and importance of plants with medicinal properties in Ghana and their usage has only been made known by some individual researchers (Addo-Fordjour et al., 2011; Boadu and Asase, 2017) through ethnobotanical means. Globally, the acceptance and utilization of herbal medicine are on a constant rise. This situation is no different in Africa where over $60 \%$ of the population depend directly on plants for their primary healthcare requirement-particularly in the developing countries. This makes plants a major contributor to natural products and forms an important part of health care. The pharmaceutical industry depends greatly on traditional medicines; thus globally, a quarter of all prescribed drugs are as a result of the extraction from these medicinal plants. Thus, medicinal plants are preferred owing to the significantly lower adverse reactions and being economical in comparison with synthetic drugs (Pushpanathan et al., 2020). Classifying plants with medicinal properties are beneficial in many ways to humans therefore it has been of importance to address this problem (Barimah and Akotia, 2015).

The use of plants as medicine has generated the need for plant identification to determine whether a particular plant is of medicinal use or not. Also, it is easy to confuse two different plants when closely looked at by untrained eyes. This makes plant identification a very crucial aspect that cannot be taken lightly in the field of natural products and medicine as misidentification can bring potentially serious consequences (Boadu and Asase, 2017). Using conventional keys (i.e., scientific names) to identify plants is complex, time-consuming, tedious for non-botanists and creates a daunting challenge for freshmen interested in acquiring specific knowledge (Wäldchen and Mäder, 2018). Using the diverse morphological characteristics of plants to distinguish between them is a challenging task. These challenges are mainly high intra-class variability and small inter-class differences (Šulc and Matas, 2017). Plant categories are closely related and often 
exhibit some level of relationship between some of their structural parts leading to low inter-class differences. Plants also show a difference in shape characteristics due to environmental influences such as climate change, topographical location etc. (Sfar et al., 2013). This makes plants broadly different in size, shape, color and texture with varying appearance throughout the year leading to high intra-class variations (Lasseck, 2017). With the swift advancement of metabolite- related databases (KNApSAcKCoreDB), data mining tools have been suggested to investigate the systematics value of metabolite-content of plants (Afendi et al., 2012).

Gabor filters are widely applied in many areas like palmprint identification (Zhang et al., 2003), fingerprint identification (Areekul et al., 2005) vehicle detection (Sun et al., 2002), facial expression (Barbu, 2010), image classification and disease detection (Qian et al., 2003; Sahebrao et al., 2015; Zheng, 2010). Gabor filters also found their application in plant identification (Cope et al., 2010; Tang et al., 2003; Venkatesh and Raghavendra, 2011) and plant disease identification (Patil and Kumar, 2017; Yang et al., 2019).

Studies have revealed two shortcomings of Gabor filters. The first concern is with the limitation on the maximum bandwidth which is set to one octave and the second issue is the presence of the nonzero DC element in the even-symmetric filter open bandwidths. Hence, Gabor filters would not be the go-to option for research that seeks to achieve broad spectral details with maximal spatial localization (Wang et al., 2008). Log-Gabor function serves as an alternative function to the Gabor function (Arrospide and Salgado, 2013).

\section{Literature Review}

\section{Plant Identification}

Plants in general are recognizable by their features such as leaves, fruits, flowers or plants as a whole. Out of all the keys of identification, the most promising and effective means of medicinal plants identification is the use of the leaves (Ab Jabal et al., 2013; De Luna et al., 2017; Lee et al., 2016; Pushpanathan et al., 2020; Wäldchen and Mäder, 2018; Yigit et al., 2019).

The term texture is used in describing an object or phenomenon surfaces. It is the essential feature used in pattern recognition in computer vision undoubtedly (Zhang et al., 2012). Texture analysis is utilized in extracting features in an image for recognition. Texture in this case, refers to an image's spatial arrangement of intensities. Thus, texture extraction becomes the quantification of the connections of the intensities' spatial arrangement. In the identification and classification of plants, the leaf texture plays a key role. Fractal Dimensions (FracDim), Gabor Filters (GF) and Gray-Level Co-occurrence Matrix (GLCM) are some popular techniques for characterization of leaf texture towards plant identification (Casanova et al., 2009; Kadir et al., 2011a). Texture analysis involves four basic methods and they are Statistical methods (First Order Statistics, Second Order Statistics, Auto correlation etc.), Geometrical methods (Morphological methods, Pattern spectrum, etc.), Model based methods (Random Mosaic Model, Autoregressive Model, Fractal Dimension, etc.) and Signal processing methods (Eigen Filters, Fourier Domain Filtering, Gabor Filter, etc.) (Armi and Fekri-Ershad, 2019). Signal processing methods used in texture characterization over time have proven a great tool (Stepień, 2014) since they are able to extract features using the first and second order statistics as well as collect the dispersal of filter responses (Nava et al., 2011).

Plant morphology studies the external textures and the physical form of plants (such as the leaf, flower, bark, stem etc.) while the studying of the internal plant structure which usually occurs at the microscopic/cellular level is referred to as plant anatomy. Classification systems in plants have seen an extensive improvement possibly owing to the morphological characteristics of plants which have become the basis and framework for advances in taxonomy. The science of chemotaxonomy aids in classifying plants using their chemical constituents. In any living organism, it is obvious that the primary metabolites produce secondary metabolites. These metabolites have a chemical structure and a biosynthetic pathway which becomes explicit and constricted to organisms that are taxonomically related and thus enhancing their classification (Lou et al., 2021).

Traditional chemosystematics of plants considers the absence or presence of various metabolites (Singh, 2016; Wink, 2003). This approach borders on the hypothesis that selected secondary metabolites dominate within a given taxon. Chemosystematics in plants has initially been used to establish the differences in other organisms and plants recognition which is to be avoided and those beneficial for food. The insight from this has gradually been made official using harmful, inactive and useful chemical constituents from significant taxa presently recognized and recorded. Plant chemosystematics could reveal the universally known natural history of plants taking cognizance of its interaction with the environment and similar plants (Christenhusz, 2020; Reynolds, 2007; Singh, 2016).

In recent years, although the chemotaxonomy approach has fast developed, yet the traditional method for classifying plants using their comparative external morphological characters remains essential to systematics and it dominates other forms of taxonomic features used in plant classification owing to reasons such as; the easily observable morphological characters; because they have innumerable variations, they assist in identification and delimitation; one is not required to obtain a sophisticated laboratory arrangement to assess these morphological characters. To 
study anatomical features of plants, one would require possibly a light microscope or a hand lens or a dissecting microscope as well as effort and time to harness information from sources such as molecular biology and photochemistry ensuring the merit of morphological characters against the other forms of plant taxonomy.

The KNApSAcKCoreDB is useful in multifaceted plant research as an extensive plant-metabolite relation DB. These researches could be on systems biology, bioinformatics, construction of integrated DBs and identification of metabolites (Ikeda et al., 2013; Nakamura et al., 2014) and also a potential source of advanced metabolites contents of plants (Shinbo et al., 2006). The KNApSAcK Family database systems have seen several usages of metabolomics studies. For example, previously the KNApSAcK Family DB systems have been deployed to appreciate the medicinal utilization of plants based on modern and traditional knowledge (Afendi et al., 2013; Wijaya et al., 2014).

There has been a study of several methods for recognizing plants by studying their leaf texture, shape, color and venation. Some of the methods include Gabor Filters (GF), Fractal Dimensions (FracDim), Gray Level Co-occurrence Matrix (GLCM), Histogram of Oriented Gradient (HoG) for leaf texture; (Backes and Bruno, 2009; Casanova et al., 2009; Cope et al., 2010; Kebapci et al., 2011; Rossatto et al., 2011; Sá et al., 2013; Syahputra et al., 2014; Zhai and Du, 2008), Simple and Morphological Shape Descriptors (SMSD), Hu moments, Fourier Descriptor (FD), Tchebichef Moment Invariant (TMI), Centroid Contour Distance (CCD), Zernike Moment Invariant (ZMI), Harmonic mean projecting transform for leaf shape; (Aakif and Khan, 2015; Chaki et al., 2015b; Du et al., 2007; Hossain and Amin, 2010; Kadir et al., 2011b; Lee and Chen, 2006; Teng et al., 2009; Zahra et al., 2020), Color Moments (CM), Color Histograms (CH), Color Co-occurrence Matrices (CCM) for leaf color; (Caglayan et al., 2013; Che Hussin et al., 2013; Kebapci et al., 2011; Prasad et al., 2013; Yanikoglu et al., 2014). Areoles morphology, Leaf vein and Run-length features for leaf venation; (Gu et al., 2005; Larese et al., 2012; Larese et al., 2014a; 2014b)

\section{Deep Learning}

An extensive family of machine learning techniques is deep learning and it is grounded on learning data observations. Deep learning architectures like convolutional Deep Neural Networks (DNN), recurrent neural networks and deep belief networks have been employed in sectors such as natural language processing, computer vision, bioinformatics, audio recognition and automatic speech recognition, having produced remarkable outcomes on several works. This concept has been categorized as a buzzword for neural networks (Benuwa et al., 2016; Gomes, 2014). For learning tasks that require supervision, applying deep learning techniques removes feature engineering via the translation of the data into compact intermediate forms similar to principal components and this minimizes redundancy in representations as it derives layered structures (Deng and $\mathrm{Yu}, 2013)$. Numerous deep learning models are used in unsupervised learning problems, thus, offering a significant advantage since untagged data is normally (generally) in abundance than tagged data. Deep belief network is one of the deep frameworks used in an unsupervised form (Bengio et al., 2013). DNN is generally interpreted in terms of the Universal approximation theorem or Probabilistic inference (Hinton et al., 2006; Murphy and Murphy, 2012).

In current years, the development of Convolutional Neural Network (CNN) has proved to be an efficient identification technique and gathered widespread attention. CNN now has become one most effective techniques in the pattern classification and image processing fields (Krizhevsky et al., 2017; Lecun et al., 2015) and is relatively preferred over conventional techniques such as the supervised and unsupervied learning techniques (Chatfield et al., 2011) by verifying on a wide scale. CNN replicates the visual cortex of humans and it's the choice of neural network for computer vision (image and video recognition). The brain's visual cortex comprises discontinuous layers of both complex and simple cells and inspires CNN modelling. CNN is also adopted in other areas such as Natural Language Processing (NLP), drug discovery, etc.

Architectural designs of CNN appear in many forms; but, generally, they constitute grouped pooling (subsampling) and convolutional in modules. The pooling layers function to minimize the feature maps' spatial resolution and so attain spatial invariance to input translations as well as input distortions (Lecun et al., 2015; Ranzato et al., 2007). The pooling layer is utilized in minimizing the spatial size or image's resolution and the number of parameters hence reducing the computation burden. This is shown by minimizing the number of links between the convolutional layers (Gu et al., 2018). There are usually alternations between the pooling layers and convolutional layers. Max pooling and Average pooling are the commonest types of pooling. Individual CNN comprises a dissimilar number of convolution layers relying on network requirements. The low level such as edges, corners are learnt by the initial convolutional layers which are passed to the other convolutional layers to acquire higher-level features. Convolutional layers functions for feature extractors, hence, the feature representations of the input images are learnt by the layers. Within a feature map, all the neurons are constricted to have their weights equalized; nonetheless, within the same convolutional layer, different feature maps possess varying weights, thus enabling the 
extraction of many features are each location (Lecun et al., 2015; Yu et al., 2014). Formally, it is computed below as the kth output feature map $Y_{k}$ :

$$
Y_{k}=f\left(W_{k} * x\right)
$$

where, " $x$ " denotes image input; " $W_{k}$ " represents the coiled filter linked to the kth feature map, the two-dimensional convolutional operator is symbolized by the multiplication $\operatorname{sign}(*)$, the inner product of the filter model is expressed separately at every location of the input image; as the nonlinear activation function is also shown by $f(\cdot)$ (Yu et al., 2014).

\section{Related Works}

Gabor filters when combined with other texture analytical techniques were found to provide better performance. Tan and Triggs (2007) extracted features from the images using Gabor and LBP and then dimensionality reduction was performed using Principal Component Analysis (PCA) method. After normalization, all the extracted features were fused and then classified.

Introduced new Gabor filter banks specifically made to identify plant species using their bark texture features. In this method, the texture was constructed as several narrowband signals separated using their normalized ratios of amplitudes and central frequencies. To integrate the narrowband signals, the normalized ratios of amplitude were used as an energy weight. This model used allowed for a collection of features of the bark texture which was obtained from every kind of plant bark assisting the design of the equivalent Gabor filter bank while differentiation of plant was made possible.

Lin et al. (2008) performed a joint analysis of Gabor filter and LBP for classification of plant leaves. A database of about 500 leaf images corresponding to 27 categories was used with a filter bank of 20 filters $(5$ scales and 4 orientations). An accuracy of $85.44 \%$ was achieved.

Casanova et al. (2009) using a Gabor bank of 64 filters (8 rotation filter and 8 scale filters) found out that Gabor filter outperformed FD and GLCM after using it on leaf lamina and margins. Other authors also found out that a combination of Gabor filter and GLCM performed better than using them individually (Chaki et al., 2015a; Cope et al., 2010). Venkatesh and Raghavendra (2011) proposed a combination of Gabor filter with the Local Binary Pattern (LBP) descriptor and found out that it performed better than using them individually. Gabor filter had a higher discriminatory power after comparing it to Histogram of Oriented Gradient for texture analysis (Yanikoglu et al., 2014).

Plant leaf identification using Gabor filters (Tang et al., 2003; Venkatesh and Raghavendra, 2011) was performed to analyse features in the spatial domain at different orientations and frequencies. Gabor filters and
Co-occurrence matrix was used in combination for plant identification by Cope et al. (2010).

Backes et al. (2009) after numerous tests performed achieved the best results of $82.93 \%$ using a family with 64 filters (eight rotations and eight scales). Patil and Bhagat (2016) used Gabor features to extract leaf texture features. Using the UCI Machine Repository Dataset, 85\% was accuracy was used whiles $94.1 \%$ accuracy was achieved using Swedish Leaf Dataset.

Cope et al. (2010) proposed a technique for plant texture classification based on joint distribution of Gabor filter responses using a filter bank of 128 filters. This method achieved excellent recognition rate of $95 \%$ on Brodatz dataset and was also effective in the case of laborious task of plant classification based on leaf analysis.

Alamoudi et al. (2020) used a number of texturebased features including Gabor and Laplacian of Gaussian filters followed by the Grey Level Co-occurrence Matrix to generate leave image features. The proposed method achieved about $93.7 \%$ accuracy. Wang et al. (2020) presented a novel counting-based leaf recognition method based on the elliptical half Gabor wavelet and maximum gap local line direction patterns. Leaf database used was the Swedish, Flavia and ICL database. The half Gabor achieved an average of $85 \%$ on all databases.

Several authors have used neural networks and also CNN in particular for plant identification. Jassmann et al. (2015) developed a mobile application for classifying plants using CNN based on the nature of the leaf using the Image CLEF data set. The architecture proposed consisted of a layer that is convoluted, followed by a composite layer and two fully connected layers applied to the $60 \times$ 80-pixel input image. Bao et al. (2019) proposed a system using two methods (Histogram of Oriented Gradient (HoG) and deep convolutional neural network) for the problems concerning identifying plants using their leaf patterns. HoG was used in classifying the features and CNN for identification purposes. Adetiba et al. (2021) leveraged on five pre-trained CNN models (Alex Net, Goog LeNet, VGG-19, ResNet50 and MobileNetV2) and Leaf snap image dataset of 185 plant species to empirically develop an accurate plant species recognition. Among the pre-trained models, MobileNetV2 with ADAM optimizer gave the highest testing accuracy of $92.33 \%$.

Even though Gabor filters provide promising results when it comes to plant identification using texture, it was seen that performance increases when Gabor filters are combined with other features like color, shape, venation and other texture feature extraction methods such as the LBP and GLCM. In (Casanova et al., 2009), using a combination of Gabor filter and Gray Level Co-occurrence Matrix (GLCM) to model the texture of plant leaf, the accuracy of the model increased form 81.6 to $97.6 \%$ when texture model was combined with shape features. Also, using a combination of Gabor filters and Local Binary 
Patterns (LBP), the accuracy of the model increased from $85 \%$ using only Gabor filters to $90 \%$ (Lin et al., 2008). In Patil and Bhagat (2016), the performance of the model also improved from $94 \%$ when using Gabor filters to $96 \%$ when combined with GLCM using the Swedish leaf dataset and 85 to $88 \%$ and using the UCI Machine Repository Dataset.

The parameters of Gabor filters which includes the filter size, standard deviation, scale and orientation influence the accuracy of the model. To achieved best results, a combination of the parameters must be investigated to provide the best results. Another limitation in using Gabor filters is that, rotation and scaling greatly influences the identification process. Therefore, Gabor filters must be defined to cover all possible orientations.

Lastly, there is a high computational cost due to using filter banks required in Gabor filters. This can be mitigated by using feature selection or reduction methods like the Principal Component Analysis (PCA).

\section{Methodology}

\section{Gabor Filter}

One of the processing signal techniques for the extraction of texture is the Gabor filter. Gabor filters are wavelets band in which individual wavelet captures energy at a particular direction and frequency. Its operation uses a local band having a known optimal localization pass filter features in both the frequency domain and the spatial domain. Gabor functions were first proposed by Dennis Gabor (Rai and Rivas, 2020). These filters have obtained immeasurable attention over the years. This is due to the fact these filters can estimate certain visual cortex cells of some mammals. Gabor filters' orientation and frequency representations are closely related to that of the human visual system and they are found to be significantly suitable for textural representation and discrimination. Also, these filters are known to have optimal localization properties present in frequency and spatial domain, hence making it best suited for problems with texture segmentation. These filters should be considered a sinusoidal plane of a certain orientation and frequency, thus Gabor filters are orientation-sensitive.

A Gabor filter has both frequency-selective and spatial-selective properties together with optimal joint resolution in both frequency and spatial domains (Munawar et al., 2021). These functions shown by the product of a sinusoid and Gaussian function constitute a single family of linear filters that behave optimally in the sense that their simultaneous resolution in both domains is maximal (Rai and Rivas, 2020). Specifically, using a Gabor filter in texture analysis was motivated due to the studies of Daugman on visual modeling of simple cells based on the experimental findings on the orientation selectivity of visual cortical neurons previously observed by Hubel and Wiesel in human beings and cats (Hubel and Wiesel, 2012; Kong, 2009).

A two-dimensional Gabor function is defined as a sinusoidal wave multiplied by a Gaussian function in a complex number form (Yuan et al., 2020):

$$
g_{\Theta}(x, y)=\exp \left(-\frac{x^{\prime 2}+\gamma^{2} y^{\prime 2}}{2 \sigma^{2}}\right) \exp \left(i\left(2 \pi \frac{x^{\prime}}{\lambda}+\phi\right)\right)
$$

Thus:

$i=$ Imaginary unit and $\Theta=\{\lambda, \theta, \phi, \sigma, \gamma\}$ is the parameter:

$$
\begin{aligned}
& x^{\prime}=x \cos (\theta)+y \sin (\theta) \\
& y^{\prime}=-x \sin (\theta)+y \cos (\theta)
\end{aligned}
$$

There are five parameters in Gabor function and each of them has a specific meaning and every parameter can take values in a specific range:

- Orientation $(\theta)$

$\theta$ specifies the orientation of the Gabor filter generated by the Gabor function. Valid values are real number between 0 and $2 \pi$

- Wavelength $(\lambda)$

$\lambda$ represents the wavelength of the Gabor filter and its values is specified in pixels. Generally, valid values of $\lambda$ are real number equal or greater than 2

- $\quad$ Phase offset $(\varphi)$

$\varphi$ is the phase offset in the argument of the sine or cosine factor in the Gabor function. Its valid values are real numbers from $-\pi$ to $\pi$. The values 0 and $\pi$ correspond to center-symmetric 'center-on' and 'center-off' functions, respectively, while $-\pi / 2$ and $\pi / 2$ correspond to anti-symmetric functions

- Aspect ratio $(\gamma)$

$\gamma$ shows the ellipticity of the support of the Gabor function. When $\gamma<1$ the support is elongated in orientation of the parallel stripes of the function and when $\gamma=1$, the support is circular. Generally, it takes real values that are greater than 0 and less or equals than 1 , so its range is $(0,1]$

- Generate $\sigma$ from bandwidth $b$

Parameter $\sigma$ is the standard deviation of the Gaussian factor of the Gabor function. Since $\lambda$ and $\sigma$ are not independent argues that $\sigma$ cannot be rightly stated and can only be generated via $b$, the bandwidth where it satisfies $\frac{\sigma}{\lambda}=\frac{1}{\pi} \sqrt{\frac{\log 2}{2}} \frac{2^{b}+1}{2^{b}-1} \triangleq \hat{b}$

\section{Log Gabor Filter}

Gabor transforms over-represent the low-frequency components and under-represent the high-frequency components. Log-Gabor function when viewed on a 
logarithmic axis has Gaussian transfer functions having frequency-response functions synonymous in several cortical cells. Again, this filter provides an extension with null DC elements, thereby arbitrarily large bandwidths are allowed to be created. A comparison of the Log-Gabor filters with the Gabor Filters shows that as Log-Gabor filters are formed using arbitrary bandwidth with the minimal spatial extent optimization feature while permitting the reduction of over-representation of low frequencies. It is also meaningful that measurements on mammalian visual systems indicate we have cell responses that are symmetric on the log frequency scale as the Log-Gabor function. Hence, the postulation on the log-Gabor functions should have the ability to encode natural images through an enhanced representation of the higher frequency constituents if the function has extended tails at the high frequency ends. Furthermore, a log-Gabor Filter always has a null DC component and therefore, the filter bandwidth can be optimized to produce a filter with minimal spatial extent. Gaussian transfer functions can be observed in Gabor functions from the perspective of the linear frequency scale; as Log-Gabor functions also shows the same features when viewed using the logarithmic frequency scale. Owing to the individuality in the log function at origin, a two-dimensional Log-Gabor filter is built in the frequency domain. Using polar coordinates system divides the filters into two components: The angular filter and the radial filter.

The frequency response of the angular filter is given by:

$$
G_{\theta}(\theta)=\exp \left(-\frac{\left.\left[\theta-\theta_{0}\right)\right]^{2}}{2 . \sigma_{\theta}^{2}}\right)
$$

and the frequency response of the radial filter can be described by:

$G_{r}(r)=\exp \left(-\frac{\left[\log (r / f)_{0}\right]^{2}}{2 . \sigma_{r}^{2}}\right)$
The two components are multiplied together to construct the overall Log-Gabor filter which has the transfer function as:

$G(r, \theta)=G_{r}(r) \cdot G_{\theta}(\theta)$

where, $\sigma_{\theta}$ determines the angular bandwidth, $\sigma_{r}$ determines the scale bandwidth, $\theta_{0}$ is the orientation angle of the filter, $f_{0}$ is the center frequency of the filter and $(r, \theta)$ represents the polar coordinates.

The frequency response of log-Gabor filters in polar coordinates is given by (Nixon and Aguado, 2020):

$L G_{m, n}(f, \theta)=\exp \left\{-\frac{\left(\log \left(f / F_{m}\right)\right)^{2}}{2(\log \beta)^{2}}\right\} \exp \left\{-\frac{\theta-\theta_{n}}{2 \sigma_{\theta}^{2}}\right\}$

The proposed framework is seen in Fig. 1.

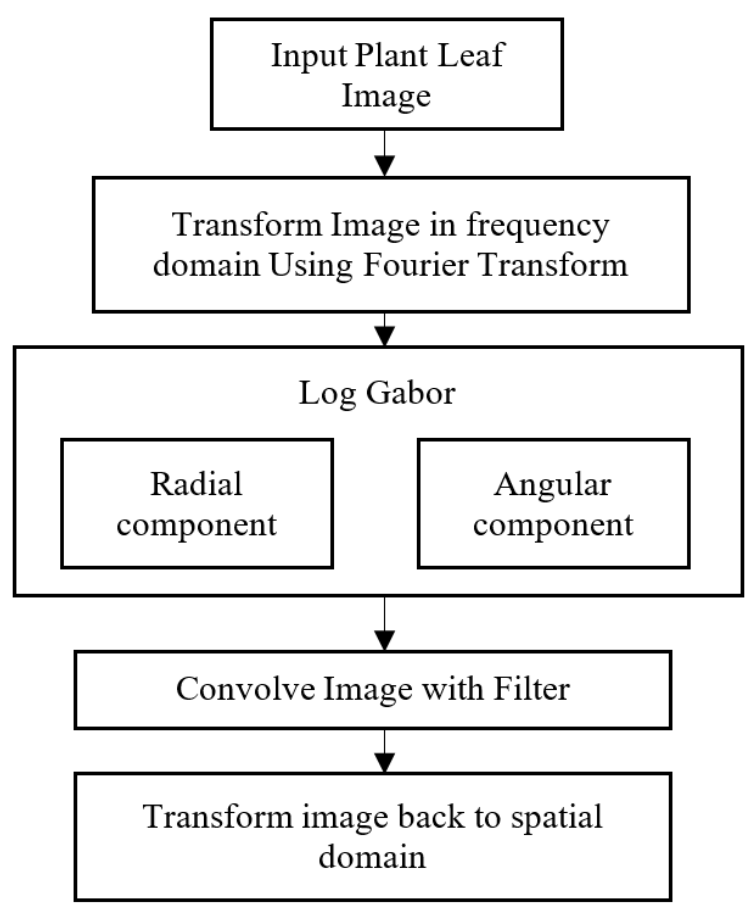

Fig. 1: Proposed framework

Table 1: Areas gabor filters have been applied

\begin{tabular}{ll}
\hline Area & References \\
\hline Palmprint identification & Zhang et al. $(2003)$ \\
Fingerprint identification & Areekul et al. $(2005)$ \\
Vehicle detection & Sun et al. $(2002)$ \\
Facial expression & Barbu (2010) \\
Image classification and disease detection & Qian et al., (2003); Zheng, (2010) \\
Plant identification & Cope et al. (2010); Tang et al., (2003); \\
& Venkatesh and Raghavendra, (2011) \\
Plant disease identification & Patil and Kumar (2017); Yang et al., (2019) \\
\hline
\end{tabular}


Table 2: Various methods for plant leaf classification

\begin{tabular}{lll}
\hline Feature & Method & References \\
\hline Texture & Gabor Filters (GF), Fractal Dimensions (FracDim), & (Backes and Bruno 2009; Casanova et al., 2009; Cope et al., \\
& Gray Level Co-occurrence Matrix (GLCM), Histogram & 2010; Kebapci et al., 2011; Rossatto et al., 2011; Sá et al., \\
& of Oriented Gradient (HoG) & 2013; Syahputra et al., 2014; Zhai and Du, 2008) \\
Shape & Simple and Morphological Shape Descriptors(SMSD), & (Aakif and Khan, 2015; Chaki et al., 2015b; Du et al., 2007; \\
& Hu moments, Fourier Descriptor (FD), Tchebichef Moment & Hossain and Amin, 2010; Kadir, Nugroho et al., 2011; Lee \\
& Invariant (TMI), Centroid Contour Distance (CCD), Zernike & and Chen, 2006; Teng et al., 2009; Zahra et al., 2020) \\
& Moment Invariant (ZMI), Harmonic Mean Projecting transform & \\
Color & Color Moments (CM), Color Histograms (CH), Color & (Caglayan et al., 2013; Che Hussin et al., 2013; Kebapci et al., \\
& Co-occurrence Matrices (CCM) & 2010; Prasad et al., 2013; Yanikoglu et al., 2014). \\
Venation & Areoles morphology, Leaf vein and Run-length features & (Gu et al., 2005; Larese et al., 2012; Larese et al., 2014a; 2014b) \\
\hline
\end{tabular}

Table 3: Summary of reviewed papers

\begin{tabular}{|c|c|c|c|c|}
\hline No. & Dataset & Filter bank & Accoracy & Reference \\
\hline 1 & The Pl@ant Leaves dataset & 64 filters & $43.66 \%$ & Casanova et al. (2009) \\
\hline 2 & N/A & 64 filters & $82.93 \%$ & Backes et al. (2009) \\
\hline 3 & Brodatz dataset & 128 filters & $85.16 \%$. & Cope et al. (2010) \\
\hline 4 & Flavia dataset & N/A & $87.1 \%$ & Chaki et al. (2015a) \\
\hline 5 & UCI machine repository dataset, swedish leaf dataset & N/A & $94 \%$ & Patil and Bhagat (2016) \\
\hline 6 & Swedish, Flavia and ICL dataset & N/A & 85.73 & Wang et al. (2020) \\
\hline 7 & N/A & N/A & $93.7 \%$ & Alamoudi et al. (2020) \\
\hline
\end{tabular}

\section{Analysis}

Gabor filters are widely applied in many areas as shown in Table 1. There has been a study of several methods for recognizing plants by studying their leaf texture, shape, color and venation as shown in Table 2. A summary f reviewed indicates Gabor filters generally have a high accuracy rate. The dataset commonly used were the UCI Machine Repository Dataset, Swedish Leaf, ICL dataset and The Pl@ant Leaves dataset. Lastly, a filter bank of between 64 and 128 were used most often.

\section{Conclusion}

Automated identification of plant species is a subject propelled by researchers who have expertise in computer learning, computer vision and multimedia information retrieval. Even though the shape of a leaf is the most discriminative or more dominant character for leaf classification, the texture also has high importance since it can reveal or capture complementary information. Additionally, plants can be easily identified just with a portion of the leaf without the entire shape or color of the leaf using leaf texture analysis. Hence, researchers and botanists can benefit greatly from texture analysis in identifying damaged plants. Specifically, the texture depicts leaf venation details and other eventual directional features and predominately permits the description of micro-texture at the leaf of fine nuances.

Lastly, Gabor filters have been extensively used in plant leaf recognition but since it suffers form limitation on the maximum bandwidth and also the presence of the nonzero DC element in the even-symmetric filter. Gabor filters would not be the go-to option for research that seeks to achieve broad spectral details with maximal spatial localization. This limitations are addressed by Log-Gabor filters, therefore can be used to improve accuracy for plant identification.

In the future work, the approach can be fused with Convolution Neural Networks to exploit the advantages of deep learning techniques.

\section{Author's Contributions}

Stephen Opoku Oppong: Collected analyzed the papers for the review and drafted the manuscript.

Frimpong Twum: Verified and supervised the whole project.

James Ben Hayfron-Acquah and Yaw Marfo Missah: Reviewed the manuscript for significant intellectual content.

\section{Ethics}

The corresponding author confirms that all the other authors have read and approved the manuscript and no ethical issues involved.

\section{References}

Aakif, A., \& Khan, M. F. (2015). Automatic classification of plants based on their leaves. Biosystems Engineering, 139, 66-75. doi.org/10.1016/j.biosystemseng.2015.08.003

Ab Jabal, M. F., Hamid, S., Shuib, S., \& Ahmad, I. (2013). Leaf features extraction and recognition approaches to classify plant. Journal of Computer Science, 9(10), 1295-1304. doi.org/10.3844/jcssp.2013.1295.1304 
Addo-Fordjour, P., Anning, A. K., Akanwariwiak, W. G., Belford, E. J. D., \& Firempong, C. K. (2011). Medicinal plants of Ghana. Genetic Resources, Chromosome Engineering and Crop Improvement: Medicinal Plants, 6, 221-246. doi.org/10.1201/b11177-14

Adeniyi, A., Asase, A., Ekpe, P. K., Asitoakor, B. K., Adu-Gyamfi, A., \& Avekor, P. Y. (2018). Ethnobotanical study of medicinal plants from Ghana; confirmation of ethnobotanical uses and review of biological and toxicological studies on medicinal plants used in Apra Hills Sacred Grove. Journal of Herbal Medicine, 14(October 2016), 76-87. doi.org/10.1016/j.hermed.2018.02.001

Adetiba, E., Ajayi, O. T., Kala, J. R., Badejo, J. A., Ajala, S., \& Abayomi, A. (2021). LeafsnapNet: An Experimentally Evolved Deep Learning Model for Recognition of Plant Species based on Leafsnap Image Dataset. Journal of Computer Science, 17(3), 349-363. doi.org/10.3844/JCSSP.2021.349.363

Afendi, F. M., Okada, T., Yamazaki, M., Nakamura, Y., Nakamura, K., Ikeda, S., Takahashi, H., \& Kanaya, S. (2012). KNApSAcK Metabolite. 1. doi.org/10.1093/pcp/pcr165.Panel

Afendi, F. M., Ono, N., Nakamura, Y., Nakamura, K., Darusman, L. K., Kibinge, N., Morita, A. H., Tanaka, K., Horai, H., Altaf-Ul-Amin, M., \& Kanaya, S. (2013). Data mining methods for omics and knowledge of crude medicinal plants toward big data biology. Computational and Structural Biotechnology Journal, 4(5), e201301010. doi.org/10.5936/csbj.201301010

Alamoudi, S., Hong, X., \& Wei, H. (2020). Plant Leaf Recognition Using Texture Features and SemiSupervised Spherical K-means Clustering. Proceedings of the International Joint Conference on Neural Networks, 1-8. doi.org/10.1109/IJCNN48605.2020.9207386

Areekul, V., Watchareeruetai, U., Suppasriwasuseth, K., \& Tantaratana, S. (2005). Separable gabor filter realization for fast fingerprint enhancement. Proceedings - International Conference on Image Processing, ICIP, 3, 253-256. doi.org/10.1109/ICIP.2005.1530376

Armi, L., \& Fekri-Ershad, S. (2019). Texture image analysis and texture classification methods - A review. 2(1), 1-29. http://arxiv.org/abs/1904.06554

Arrospide, J., \& Salgado, L. (2013). Log-gabor filters for image-based vehicle verification. IEEE Transactions on Image Processing, 22(6), 2286-2295. doi.org/10.1109/TIP.2013.2249080
Backes, A. R., \& Bruno, O. M. (2009). Plant leaf identification using multi-scale fractal dimension. International Conference on Image Analysis and Processing, 5716 LNCS, 143-150. doi.org/10.1007/978-3-642-04146-4_17

Backes, A. R., Casanova, D., \& Bruno, O. M. (2009). Plant leaf identification based on volumetric fractal dimension. International Journal of Pattern Recognition and Artificial Intelligence, 23(6), 1145-1160. doi.org/10.1142/S0218001409007508

Bao, T. Q., Kiet, N. T. T., Dinh, T. Q., \& Hiep, H. X. (2020). Plant species identification from leaf patterns using histogram of oriented gradients feature space and convolution neural networks. Journal of Information and Telecommunication, 4(2), 140-150. doi.org/10.1080/24751839.2019.1666625

Barbu, T. (2010). Gabor filter-based face recognition technique. Proceedings of the Romanian Academy Series A - Mathematics Physics Technical Sciences Information Science, 11(3), 277-283.

Barimah, K. B., \& Akotia, C. S. (2015). The promotion of traditional medicine as enactment of community psychology in Ghana. Journal of Community Psychology, 43(1), 99-106. doi.org/10.1002/jcop.21687

Bengio, Y., Courville, A., \& Vincent, P. (2013). Representation learning: A review and new perspectives. IEEE Transactions on Pattern Analysis and Machine Intelligence, 35(8), 1798-1828. doi.org/10.1109/TPAMI.2013.50

Benuwa, B.-B., Ghansah, B., \& Keddy, D. (2016). Review of Complex Networks. International Journal of Computer Applications, 141(5), 33-43. doi.org/10.5120/ijca2016909624

Boadu, A. A., \& Asase, A. (2017). Documentation of herbal medicines used for the treatment and management of human diseases by some communities in southern Ghana. Evidence-Based Complementary and Alternative Medicine, 2017. doi.org/10.1155/2017/3043061

Caglayan, A., Guclu, O., \& Can, A. B. (2013). A plant recognition approach using shape and color features in leaf images. In Lecture Notes in Computer Science (including subseries Lecture Notes in Artificial Intelligence and Lecture Notes in Bioinformatics): Vol. 8157 LNCS (Issue PART 2, pp. 161-170). Springer Berlin Heidelberg. doi.org/10.1007/978-3-642-41184-7_17

Casanova, D., De Mesquita Sá, J. J., \& Bruno, O. M. (2009). Plant leaf identification using gabor wavelets. International Journal of Imaging Systems and Technology, 19(3), 236-243. doi.org/10.1002/ima.20201 
Chaki, J., Parekh, R., \& Bhattacharya, S. (2015a). Plant leaf recognition using texture and shape features with neural classifiers. Pattern Recognition Letters, 58, 61-68. doi.org/10.1016/j.patrec.2015.02.010

Chaki, J., Parekh, R., \& Bhattacharya, S. (2015b). Recognition of whole and deformed plant leaves using statistical shape features and neuro-fuzzy classifier. 2015 IEEE 2nd International Conference on Recent Trends in Information Systems, ReTIS 2015 - Proceedings, 189-194. doi.org/10.1109/ReTIS.2015.7232876

Chatfield, K., Lempitsky, V., Vedaldi, A., \& Zisserman, A. (2011). The devil is in the details: An evaluation of recent feature encoding methods. Procedings of the British Machine Vision Conference 2011, 76.176.12. doi.org/10.5244/c.25.76

Che Hussin, N. A., Jamil, N., Nordin, S., \& Awang, K. (2013). Plant species identification by using scale invariant feature transform (SIFT) and grid based colour moment (GBCM). 2013 IEEE Conference on Open Systems, ICOS 2013, 226-230. doi.org/10.1109/ICOS.2013.6735079

Christenhusz, M. J. M. (2020). On species concepts, phylogenetics and the science of natural historythree current issues facing taxonomy. Megataxa, 1(1), 67-72. doi.org/10.11646/megataxa.1.1.14

Cope, J. S., Remagnino, P., Barman, S., \& Wilkin, P. (2010). Plant texture classification using gabor cooccurrences. In Lecture Notes in Computer Science (including subseries Lecture Notes in Artificial Intelligence and Lecture Notes in Bioinformatics): Vol. 6454 LNCS (Issue PART 2, pp. 669-677). Springer Berlin Heidelberg. doi.org/10.1007/978-3-642-17274-8_65

De Luna, R. G., Baldovino, R. G., Cotoco, E. A., De Ocampo, A. L. P., Valenzuela, I. C., Culaba, A. B., \& Gokongwei, E. P. D. (2017). Identification of philippine herbal medicine plant leaf using artificial neural network. HNICEM 2017 - 9th International Conference on Humanoid, Nanotechnology, Information Technology, Communication and Control, Environment and Management, 2018-Janua, 1-8. doi.org/10.1109/HNICEM.2017.8269470

Deng, L., \& Yu, D. (2013). Deep learning: Methods and applications. Foundations and Trends in Signal Processing, 7(3-4), 197-387. doi.org/10.1561/2000000039

Du, J. X., Wang, X. F., \& Zhang, G. J. (2007). Leaf shape based plant species recognition. Applied Mathematics and Computation, 185(2), 883-893. doi.org/10.1016/j.amc.2006.07.072

Gomes, B. L. (2014, October 20). Machine-Learning Maestro Michael Jordan on the Delusions of Big Data and Other Huge Engineering Efforts What the Turing Test Really Means Why We Should Stop Using Brain Metaphors When We Talk About Computing. EEE Spectrum, Online Article, 1-10.
Gu, J., Wang, Z., Kuen, J., Ma, L., Shahroudy, A., Shuai, B., Liu, T., Wang, X., Wang, G., Cai, J., \& Chen, T. (2018). Recent advances in convolutional neural networks. Pattern Recognition, 77, 354-377. doi.org/10.1016/j.patcog.2017.10.013

Gu, X., Du, J. X., \& Wang, X. F. (2005). Leaf recognition based on the combination of wavelet transform and gaussian interpolation. In Lecture Notes in Computer Science (including subseries Lecture Notes in Artificial Intelligence and Lecture Notes in Bioinformatics): Vol. 3644 LNCS (pp. 253-262). Springer Berlin Heidelberg. doi.org/10.1007/11538059_27

Hinton, G. E., Osindero, S., \& Teh, Y. W. (2006). A fast learning algorithm for deep belief nets. Neural Computation, 18(7), 1527-1554. doi.org/10.1162/neco.2006.18.7.1527

Hossain, J., \& Amin, M. A. (2010). Leaf shape identification based plant biometrics. Proceedings of 2010 13th International Conference on Computer and Information Technology, ICCIT 2010, 458-463. doi.org/10.1109/ICCITECHN.2010.5723901

Hubel, D. H., \& Wiesel, T. N. (2012). Brain and Visual Perception: The Story of a 25-year Collaboration. In Brain and Visual Perception: The Story of a 25-year Collaboration. Oxford University Press. doi.org/10.1093/acprof:oso/9780195176186.001.0001

Ikeda, S., Abe, T., Nakamura, Y., Kibinge, N., Hirai Morita, A., Nakatani, A., Ono, N., Ikemura, T., Nakamura, K., Altaf-Ul-Amin, M., \& Kanaya, S. (2013). Systematization of the protein sequence diversity in enzymes related to secondary metabolic pathways in plants, in the context of big data biology inspired by the KNApSAcK motorcycle database. Plant and Cell Physiology, 54(5), 711-727. doi.org/10.1093/pcp/pct041

Jassmann, T. J., Tashakkori, R., \& Parry, R. M. (2015). Leaf classification utilizing a convolutional neural network. Conference Proceedings - IEEE SOUTHEASTCON, 2015-June(June). doi.org/10.1109/SECON.2015.7132978

Kadir, A., E. Nugroho, L., Susanto, A., \& Santosa, P. I. (2011a). Neural Network Application on Foliage Plant Identification. International Journal of Computer Applications, 29(9), 15-22. doi.org/10.5120/3592-4981

Kadir, A., Nugroho, L.., Susanto, A., \& Santosa, P. (2011b). A Comparative Experiment of Several Shape Methods in Recognizing Plants. International Journal of Computer Science and Information Technology, 3(3), 256-273. doi.org/10.5121/ijcsit.2011.3318

Kebapci, H., Yanikoglu, B., \& Unal, G. (2011). Plant image retrieval using color, shape and texture features. Computer Journal, 54(9), 1475-1490. doi.org/10.1093/comjnl/bxq037 
Kong, A. W. K. (2009). An analysis of gabor detection. In Lecture Notes in Computer Science (including subseries Lecture Notes in Artificial Intelligence and Lecture Notes in Bioinformatics): Vol. 5627 LNCS (pp. 64-72). Springer Berlin Heidelberg. doi.org/10.1007/978-3-642-02611-9_7

Krizhevsky, A., Sutskever, I., \& Hinton, G. E. (2017). ImageNet classification with deep convolutional neural networks. Communications of the ACM, 60(6), 84-90. doi.org/10.1145/3065386

Larese, M. G., Bayá, A. E., Craviotto, R. M., Arango, M. R., Gallo, C., \& Granitto, P. M. (2014a). Multiscale recognition of legume varieties based on leaf venation images. Expert Systems with Applications, 41(10), 4638-4647. doi.org/10.1016/j.eswa.2014.01.029

Larese, M. G., Namías, R., Craviotto, R. M., Arango, M. R., Gallo, C., \& Granitto, P. M. (2014b). Automatic classification of legumes using leaf vein image features. Pattern Recognition, 47(1), 158-168. doi.org/10.1016/j.patcog.2013.06.012

Larese, M. G., Craviotto, R. M., Arango, M. R., Gallo, C., \& Granitto, P. M. (2012). Legume identification by leaf vein images classification. In Lecture Notes in Computer Science (including subseries Lecture Notes in Artificial Intelligence and Lecture Notes in Bioinformatics): Vol. 7441 LNCS (pp. 447-454). Springer Berlin Heidelberg. doi.org/10.1007/978-3-642-33275-3_55

Lasseck, M. (2017). Image-based plant species identification with deep Convolutional Neural Networks. CEUR Workshop Proceedings, 1866.

Lecun, Y., Bengio, Y., \& Hinton, G. (2015). Deep learning. Nature, 521(7553), 436-444. doi.org/10.1038/nature14539

Lee, C. L., \& Chen, S. Y. (2006). Classification of leaf images. International Journal of Imaging Systems and Technology, 16(1), 15-23. doi.org/10.1002/ima.20063

Lee, S. H., Chang, Y. L., Chan, C. S., \& Remagnino, P. (2016). Plant identification system based on a convolutional neural network for the lifeclef 2016 plant classification task. CEUR Workshop Proceedings, 1609, 502-510.

Lin, F. Y., Zheng, C. H., Wang, X. F., \& Man, Q. K. (2008). Multiple classification of plant leaves based on gabor transform and LBP operator. In Communications in Computer and Information Science (Vol. 15, pp. 432-439). Springer Berlin Heidelberg. doi.org/10.1007/978-3-540-85930-7_55

Lou, H., Hu, L., Lu, H., Wei, T., \& Chen, Q. (2021). Metabolic engineering of microbial cell factories for biosynthesis of flavonoids: A review. Molecules, 26(15). doi.org/10.3390/molecules26154522
Munawar, H. S., Aggarwal, R., Qadir, Z., Khan, S. I., Kouzani, A. Z., \& Mahmud, M. A. P. (2021). A gabor filter-based protocol for automated image-based building detection. Buildings, 11(7), 1-15. doi.org/10.3390/buildings11070302

Murphy, P. K., \& Murphy, K. P. (2012). A probabilistic perspective. In Chance encounters: Probability in .... MIT Press.

http://link.springer.com/chapter/10.1007/978-94011-3532-0_2

Nakamura, Y., Mochamad Afendi, F., Kawsar Parvin, A., Ono, N., Tanaka, K., Hirai Morita, A., Sato, T., Sugiura, T., Altaf-Ul-Amin, M., \& Kanaya, S. (2014). KNApSAcK metabolite activity database for retrieving the relationships between metabolites and biological activities. Plant and Cell Physiology, 55(1), e7--e7. doi.org/10.1093/pcp/pct176

Nava, R., Escalante-Ramírez, B., \& Cristóbal, G. (2011, September). A comparison study of Gabor and logGabor wavelets for texture segmentation. In 2011 7th International Symposium on Image and Signal Processing and Analysis (ISPA) (pp. 189-194). IEEE. https://ieeexplore.ieee.org/abstract/document/6046604/

Nixon, M. S., \& Aguado, A. S. (2020). High-level feature extraction: Fixed shape matching. In Feature Extraction and Image Processing for Computer Vision (pp. 223-290). Elsevier. doi.org/10.1016/b978-0-12-814976-8.00005-1

Patil, A., \& Bhagat, K. S. (2016). Plants Identification by Leaf Shape using GLCM, Gabor Wavelets and PCA. International Journal of Engineering Trends and Technology, 37(3), 140-143. doi.org/10.14445/22315381/ijett-v37p222

Patil, J. K., \& Kumar, R. (2017). Analysis of content based image retrieval for plant leaf diseases using color, shape and texture features. Engineering in Agriculture, Environment and Food, 10(2), 69-78. doi.org/10.1016/j.eaef.2016.11.004

Prasad, S., Peddoju, S. K., \& Ghosh, D. (2013). Mobile plant species classification: A low computational aproach. 2013 IEEE 2nd International Conference on Image Information Processing, IEEE ICIIP 2013, 405-409. doi.org/10.1109/ICIIP.2013.6707624

Pushpanathan, K., Hanafi, M., Mashohor, S., \& Fazlil Ilahi, W. F. (2020). Machine learning in medicinal plants recognition: A review. Artificial Intelligence Review. doi.org/10.1007/s10462-020-09847-0

Qian, Z., Montillo, A., Metaxas, D. N., \& Axel, L. (2003). Segmenting Cardiac MRI Tagging Lines using Gabor Filter Banks. Annual International Conference of the IEEE Engineering in Medicine and Biology - Proceedings, 1, 630-633. doi.org/10.1109/iembs.2003.1279834 
Rai, M., \& Rivas, P. (2020). A Review of Convolutional Neural Networks and Gabor Filters in Object Recognition. Proceedings - 2020 International Conference on Computational Science and Computational Intelligence, CSCI 2020, 1560-1567. doi.org/10.1109/CSCI51800.2020.00289

Ranzato, M., Huang, F. J., Boureau, Y. L., \& LeCun, Y. (2007, June). Unsupervised learning of invariant feature hierarchies with applications to object recognition. Proceedings of the IEEE Computer Society Conference on Computer Vision and Pattern Recognition. doi.org/10.1109/CVPR.2007.383157

Reynolds, T. (2007). The evolution of chemosystematics. Phytochemistry, 68(22-24), 2887-2895. doi.org/10.1016/j.phytochem.2007.06.027

Rossatto, D. R., Casanova, D., Kolb, R. M., \& Bruno, O. M. (2011). Fractal analysis of leaf-texture properties as a tool for taxonomic and identification purposes: A case study with species from Neotropical Melastomataceae (Miconieae tribe). Plant Systematics and Evolution, 291(1), 103-116. doi.org/10.1007/s00606-010-0366-2

Sá, J. J. D. M., Backes, A. R., \& Cortez, P. C. (2013). Plant leaf classification using color on a gravitational approach. In Lecture Notes in Computer Science (including subseries Lecture Notes in Artificial Intelligence and Lecture Notes in Bioinformatics): Vol. 8048 LNCS (Issue PART 2, pp. 258-265). Springer Berlin Heidelberg. doi.org/10.1007/978-3642-40246-3_32

Sahebrao, R., N., S., T., S., \& Dhopeshwarkar, M. (2015). Automated Diagnosis Non-proliferative Diabetic Retinopathy in Fundus Images using Support Vector Machine. International Journal of Computer Applications, 125(15), 7-10. doi.org/10.5120/ijca2015905968

Sfar, A. R., Boujemaa, N., \& Geman, D. (2013). Vantage feature frames for fine-grained categorization. Proceedings of the IEEE Computer Society Conference on Computer Vision and Pattern Recognition, 835-842. doi.org/10.1109/CVPR.2013.113

Shinbo, Y., Nakamura, Y., Altaf-Ul-Amin, M., Asahi, H., Kurokawa, K., Arita, M., Saito, K., Ohta, D., Shibata, D., \& Kanaya, S. (2006). KNApSAcK: A comprehensive species-metabolite relationship database. In Biotechnology in Agriculture and Forestry (Vol. 57, pp. 165-181). Springer-Verlag. doi.org/10.1007/3-540-29782-0_13

Simpson, M. G. (2019). Plant Anatomy and Physiology. In Plant Systematics (pp. 537-566). Elsevier. doi.org/10.1016/b978-0-12-812628-8.50010-9

Singh, R. (2016). Chemotaxonomy: A Tool for Plant Classification. Journal of Medicinal Plants Studies, 4(2), 90-93.
Stepień, K. (2014). Research on a surface texture analysis by digital signal processing methods. Tehnicki Vjesnik, 21(3), 485-493.

Šulc, M., \& Matas, J. (2017). Fine-grained recognition of plants from images. Plant Methods, 13(1). doi.org/10.1186/s13007-017-0265-4

Sun, Z., Bebis, G., \& Miller, R. (2002). On-road vehicle detection using Gabor filters and support vector machines. International Conference on Digital Signal Processing, DSP, 2, 1019-1022. doi.org/10.1109/ICDSP.2002.1028263

Syahputra, H., Harjoko, A., Wardoyo, R., \& Pulungan, R. (2014). Plant recognition using stereo leaf image using gray-level co-occurrence matrix. Journal of Computer Science, 10(4), 697-704. doi.org/10.3844/jcssp.2014.697.704

Tan, X., \& Triggs, B. (2007). Fusing gabor and LBP feature sets for kernel-based face recognition. In Lecture Notes in Computer Science (including subseries Lecture Notes in Artificial Intelligence and Lecture Notes in Bioinformatics): Vol. 4778 LNCS (pp. 235-249). Springer Berlin Heidelberg. doi.org/10.1007/978-3-540-75690-3_18

Tang, L., Tian, L., \& Steward, B. L. (2003). Classification of Broadleaf and Grass Weeds Using Gabor Wavelets and an Artificial Neural Network. Transactions of the American Society of Agricultural Engineers, 46(4), 1247-1254. doi.org/10.13031/2013.13944

Teng, C. H., Kuo, Y. T., \& Chen, Y. S. (2009). Leaf segmentation, its 3D position estimation and leaf classification from a few images with very close viewpoints. In Lecture Notes in Computer Science (including subseries Lecture Notes in Artificial Intelligence and Lecture Notes in Bioinformatics): Vol. 5627 LNCS (pp. 937-946). Springer Berlin Heidelberg. doi.org/10.1007/978-3-642-02611-9_92

Venkatesh, S. K., \& Raghavendra, R. (2011). Local Gabor Phase Quantization scheme for robust leaf classification. Proceedings - 2011 3rd National Conference on Computer Vision, Pattern Recognition, Image Processing and Graphics, NCVPRIPG 2011, 211-214. doi.org/10.1109/NCVPRIPG.2011.52

Wäldchen, J., \& Mäder, P. (2018). Plant Species Identification Using Computer Vision Techniques: A Systematic Literature Review. In Archives of Computational Methods in Engineering (Vol. 25, Issue 2). Springer Netherlands. doi.org/10.1007/s11831-016-9206-Z

Wang, W., Li, J., Huang, F., \& Feng, H. (2008). Design and implementation of Log-Gabor filter in fingerprint image enhancement. Pattern Recognition Letters, 29(3), 301-308. doi.org/10.1016/j.patrec.2007.10.004 
Wang, X., Du, W., Guo, F., \& Hu, S. (2020). Leaf Recognition Based on Elliptical Half Gabor and Maximum Gap Local Line Direction Pattern. IEEE Access, 8, 39175-39183.

doi.org/10.1109/ACCESS.2020.2976117

Wijaya, S. H., Husnawati, H., Afendi, F. M., Batubara, I., Darusman, L. K., Altaf-Ul-Amin, M., Sato, T., Ono, N., Sugiura, T., \& Kanaya, S. (2014). Supervised clustering based on DPClusO: Prediction of plant-disease relations using Jamu formulas of KNApSAcK database. BioMed Research International, 2014, 1-15. doi.org/10.1155/2014/831751

Wink, M. (2003). Evolution of secondary metabolites from an ecological and molecular phylogenetic perspective. Phytochemistry, 64(1), 3-19. doi.org/10.1016/S0031-9422(03)00300-5

Yang, N., Yuan, M., Wang, P., Zhang, R., Sun, J., \& Mao, H. (2019). Tea diseases detection based on fast infrared thermal image processing technology. Journal of the Science of Food and Agriculture, 99(7), 3459-3466. doi.org/10.1002/jsfa.9564

Yanikoglu, B., Aptoula, E., \& Tirkaz, C. (2014). Automatic plant identification from photographs. Machine Vision and Applications, 25(6), 1369-1383. doi.org/10.1007/s00138-014-0612-7

Yigit, E., Sabanci, K., Toktas, A., \& Kayabasi, A. (2019). A study on visual features of leaves in plant identification using artificial intelligence techniques. Computers and Electronics in Agriculture, 156(June 2018), 369-377.

doi.org/10.1016/j.compag.2018.11.036
Yu, D., Wang, H., Chen, P., \& Wei, Z. (2014). Mixed pooling for convolutional neural networks. In Lecture Notes in Computer Science (including subseries Lecture Notes in Artificial Intelligence and Lecture Notes in Bioinformatics) (Vol. 8818, pp. 364-375). Springer International Publishing. doi.org/10.1007/978-3-319-11740-9_34

Yuan, Y., Zhang, J., \& Wang, Q. (2020). Deep Gabor convolution network for person re-identification. Neurocomputing, 378, 387-398. doi.org/10.1016/j.neucom.2019.10.083

Zahra, S. J., Sulaiman, R., Kahaki, S. M. M., \& Prabuwono, A. S. (2020). Harmonic mean projection shape transform for leaf classification. Journal of Computer Science, 16(9), 1212-1219. doi.org/10.3844/JCSSP.2020.1212.1219

Zhai, C. M., \& Du, J. X. (2008). Applying Extreme Learning Machine to plant species identification. Proceedings of the 2008 IEEE International Conference on Information and Automation, ICIA 2008, 879-884. doi.org/10.1109/ICINFA.2008.4608123

Zhang, D., Kong, W. K., You, J., \& Wong, M. (2003). Online palmprint identification. IEEE Transactions on Pattern Analysis and Machine Intelligence, 25(9), 1041-1050. doi.org/10.1109/TPAMI.2003.1227981

Zhang, J., \& Wang, G. (2012). Image matching using a bat algorithm with mutation. Applied Mechanics and Materials, 203, 88-93. doi.org/10.4028/www.scientific.net/AMM.203.88

Zheng, Y. (2010). Breast cancer detection with gabor features from digital Mammograms. Algorithms, 3(1), 44-62. doi.org/10.3390/a3010044 